\title{
Relationship between maternal body mass index with the onset of breastfeeding and its associated problems: an online survey
}

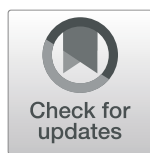

Ana Ballesta-Castillejos ${ }^{1}$, Juan Gomez-Salgado ${ }^{2,3}$, Julian Rodriguez-Almagro ${ }^{4 *}$ D, Inmaculada Ortiz-Esquinas ${ }^{5}$ and Antonio Hernandez-Martinez ${ }^{4,5}$

\begin{abstract}
Background: Obesity is a worldwide public health problem that demands significant attention. Several studies have found that maternal obesity has a negative effect on the duration of breastfeeding and delayed lactogenesis. The World Health Organization has classified Body Max Index (BMI) as normal weight (normoweight) (BMI:18.5-24.9), overweight (BMI:25-29.9), obesity grade I (30.0-34.9), obesity grade II (BMI: 35.0-39.9) and obesity grade III (BMI $\geq 40.0)$. The objective of this study is to describe the relationship between maternal BMI and breastfeeding rates, as well as breastfeeding-associated problems and discomfort in women assisted by the Spanish Health System.

Methods: To this end, a cross-sectional observational study aimed at women who have been mothers between 2013 and 2018 in Spain was developed. The data was collected through an online survey of 54 items that was distributed through lactation associations and postpartum support groups between March and June 2019. Five thousand eight hundred seventy one women answered the survey. In the data analysis, Crude Odds Ratios (OR) and Adjusted Odds Ratios (AOR) were calculated through a multivariate analysis through binary and multinomial regression.

Results: A linear relationship was observed between the highest BMI figures and the reduction of the probability of starting skin-to-skin contact (AOR for obesity type III of 0.51 [95\% Cl 0.32, 0.83]), breastfeeding in the first hour (AOR for obesity type III of 0.58 [95\% Cl 0.36, 0.94]), and exclusive breastfeeding to hospital discharge (AOR for obesity type III of $0.57[95 \% \mathrm{Cl} 0.35,0.94])$, as compared to women with normoweight.
\end{abstract}

Conclusions: Women with higher BMl are less likely to develop successful breastfeeding than women with normoweight.

Keywords: Breastfeeding, Obesity, Public health

\section{Background}

Obesity, defined as an abnormal accumulation of fat that results in excessive body weight, is usually classified through the body mass index (BMI), defined as the weight in kilograms divided by the size in square meters $(\mathrm{kg} / \mathrm{m} 2)$ [1]. The World Health Organization (WHO) has classified BMI as normoweight (18.5-24.9), overweight (25-29.9),

\footnotetext{
*Correspondence: julianj.rodriguez@uclm.es

${ }^{4}$ Department of Nursing, Ciudad Real Nursing School, University of Castilla-La Mancha, 13071 Ciudad Real, Spain

Full list of author information is available at the end of the article
}

obesity grade I (30.0-34.5), obesity grade II (35.0-39.9), and obesity grade III $(>40.0)[2,3]$.

Obesity is a worldwide public health problem that demands significant attention [4]. A recent study showed that the prevalence of obesity among pregnant women was $28.9 \%$, a trend that continues to increase [4] globally among populations in both developing and developed countries [5]. According to the Perinatal European Health Report, in most European countries, more than $30 \%$ of pregnant women are obese. The proportion of overweight or obese women ranges from 30 to $50 \%$, with

(C) The Author(s). 2020 Open Access This article is licensed under a Creative Commons Attribution 4.0 International License, which permits use, sharing, adaptation, distribution and reproduction in any medium or format, as long as you give appropriate credit to the original author(s) and the source, provide a link to the Creative Commons licence, and indicate if changes were made. The images or other third party material in this article are included in the article's Creative Commons licence, unless indicated otherwise in a credit line to the material. If material is not included in the article's Creative Commons licence and your intended use is not permitted by statutory regulation or exceeds the permitted use, you will need to obtain permission directly from the copyright holder. To view a copy of this licence, visit http://creativecommons.org/licenses/by/4.0/ The Creative Commons Public Domain Dedication waiver (http://creativecommons.org/publicdomain/zero/1.0/) applies to the data made available in this article, unless otherwise stated in a credit line to the data. 
a prevalence of less than 30\% in Croatia, Austria, and Slovenia, and around $50 \%$ in the UK [6].

Recently, the relationship between BMI and exclusive maternal breastfeeding (EMB) has been identified as less common [7], and shorter [8] in women with a higher BMI. However, this association is not a novelty given that a study carried out in 1992 by Rutishauser and Carlin reported a $50 \%$ risk of early breastfeeding discontinuation among mothers with $\mathrm{BMI}>26 \mathrm{~kg} / \mathrm{m}^{2}$ [4]. Due to the relevance of global obesity and overweight prevalence, numerous studies have focused on this issue and showed that maternal obesity has a negative effect on the duration of breastfeeding [9-11], as well as delayed lactogenesis $[9,12]$.

It is undeniable that obesity is an increasingly prevalent health problem. Specifically, in Spain, data indicate that the average population weight has increased since records exist, and if this trend continues [13], obesity rates will have increased by $16 \%$ by the year 2030 [14]. However, currently, there are no studies linking this data to newborn breastfeeding outcomes, so in this study we have considered describing the relationship between maternal BMI and breastfeeding rates, as well as breastfeedingassociated problems and discomfort in women assisted by the Spanish Health System.

\section{Methods}

\section{Design and selection of the study participants}

This is a cross-sectional observational study aimed at women who have been mothers between 2013 and 2018 in Spain. This study has received the approval of the Clinical Research Ethics Committee of Alcázar de San Juan, in Spain, with protocol number 92-C.

The participants were women over the age of 18, who understood Spanish and who agreed to fill in the questionnaire. Before filling in the questionnaire, the participants read the information sheet about the purpose of the study and give their consent to participate in it by ticking a box. After this, they were provided with the necessary information to be able to complete the questionnaire. Participants could voluntarily provide an email address or phone number through which they would be contacted in case any additional information related to the study was needed.

For the sample size estimation, the maximum modelling criterion that requires 10 events for each independent variable to be included in the multivariate model has been used [15]. For this sample calculation, the prevalence of delayed lactogenesis II has been used as the main event, which takes place in women with obesity around 58\% [9]. Considering a minimum of 15 independent variables, a minimum of 150 women with delayed lactogenesis II would be required, representing a minimum total population of 259 women under study within this scenario. However, the research team decided to include all women who met the inclusion criteria in order to obtain estimates of greater precision.

For data collection, an anonymous online questionnaire was designed with 54 items [ 47 yes/no questions and 7 multiple answer questions) on sociodemographic and obstetric variables, and on complications during pregnancy, delivery, and postpartum (Additional file 1). The questionnaire was disseminated among the Spanish lactation and postpartum support associations and groups, and those responsible for these groups were in charge of disseminating them among their members (Additional file 2).

The variables included in the study were:

The main independent variable was self-reported Body Mass Index prior to gestation, categorised normoweight (18.5-24.9), overweight (25-29.9), obesity grade I (30.0$34.9)$, obesity grade II (35.0-39.9), and obesity grade III $(\geq 40.0)$.

The independent adjustment variables were maternal age, income level, level of education, tobacco consumption, number of children, number of births, gestational age at the time of delivery, hypertension during pregnancy (high blood pressure during pregnancy), diet-controlled gestational diabetes (high sugar levels in blood during pregnancy, controlled with diet), insulin-controlled gestational diabetes (high sugar levels in blood during pregnancy, controlled with insulin), hyperthyroidism (high thyroid hormone levels), hypothyroidism (low thyroid hormone levels), anaemia (low iron levels in blood), intrahepatic cholestasis (reduction or obstruction of flow of bile in the liver), risk of preterm birth (risk of giving birth before week 37), deep vein thrombosis, oligohydramnios (decrease in amniotic fluid levels), polyhydramnios (increase in amniotic fluid levels), altered foetal heart rate (FHR) during delivery, stained amniotic fluid (AF), vaginal bleeding, nonprogression of delivery, cephalopelvic disproportion, intrapartum fever, intrapartum preeclampsia, use of epidural analgesia, use of general anaesthesia, induced delivery, end of delivery, episiotomy, tearing, low birthweight (2.500 g), macrosomia $(>4.000 \mathrm{~g})$, newborn admission, postpartumrelated subsequent maternal surgery, maternal admission into intensive care unit, and maternal re-admission. The women were asked to mark the existence of a pathology only after having been diagnosed by a physician.

In addition, 3 "composite" morbidity variables were created: pregnancy (including Hypertension during pregnancy, diet-controlled gestational diabetes, insulin-controlled gestational diabetes, hyperthyroidism, hypothyroidism, anaemia, intrahepatic cholestasis, risk of preterm birth, deep vein thrombosis); delivery (including altered foetal heart rate (FHR) during delivery, stained amniotic fluid (AF), vaginal bleeding, non-progression of delivery, cephalopelvic disproportion, intrapartum fever (body temperature higher 
than $38^{\circ} \mathrm{C}$ ), intrapartum preeclampsia, induced delivery, end of delivery by caesarean, and severe tearing (type IIIIV); neonatal (including prematurity, low birthweight (< $2.500 \mathrm{~g}$ ), macrosomia (> $4.000 \mathrm{~g}$ ), and neonatal admission to intensive care unit; and postpartum (including maternal postpartum surgery related to the delivery, maternal admission to intensive care unit, and maternal re-admission).

The dependent variables were: complications during lactation (starting skin-to-skin contact, starting breastfeeding in the first hour, and exclusive breastfeeding to hospital discharge), and complications or discomfort associated with breastfeeding during hospitalisation and at home.

\section{Statistical analysis}

First, a descriptive analysis was performed using absolute and relative frequencies for the categorical variables. A bivariate analysis was then performed including the main sociodemographic, obstetric, neonatal, lactation and BMI characteristics at the time of delivery; this was done by using the Pearson's chi-squared test with lineal trend. The analysis was performed to detect potentially confounding variables between the main dependent variables related to breastfeeding and BMI. In the next step, a multivariate analysis was carried out using binary logistic regression (through the ENTER procedure), where all the variables that could potentially be related to BMI were incorporated. Then, the Crude Odds Ratios (OR) and Adjusted Odds Ratios (AOR) were calculated with their respective 95\% confidence intervals (95\% CI) to determine the relationship between BMI and complications during breastfeeding (starting skin-to-skin contact, starting breastfeeding in the first hour, and exclusive breastfeeding to hospital discharge) and complications or discomfort both during hospitalisation and at home. All analyses were performed using the SPSS v24.0 statistical package.

\section{Results}

The studied population was 5871 women. Of these, $27.3 \%$ (1607) had normoweight, 46.4\% (2722) overweight, 19.6\% (1153) obesity type I, 5.0\% (293) obesity type II, and $0.13 \%$ (96) obesity type III. 56.1\% (3126) were primiparous, $2.4 \%$ (142) had twin pregnancy, $6.9 \%$ (405) were preterm births (<37 weeks), and $60.0 \%$ (3256) had an eutocic delivery.

A bivariate analysis was then performed to determine the sociodemographic, obstetric, neonatal, lactation and BMI characteristics at the time of delivery.

As for the relationship between BMI and the most important sociodemographic characteristics, a statistical association with maternal age, academic level, family income level, smoking habit, and attendance to childbirth was found. However, we did not find any statistically significant difference with respect to nationality and maternity classes. All the details of this analysis can be found in Table 1 .

When analysing obstetric factors in relation to BMI, the relationship between BMI and the number of births, twin pregnancies, prematurity, hypertensive pregnancy disorders, gestational diabetes (both controlled with insulin and through diet), the risk of preterm birth, deep vein thrombosis, oligohydramnios, and polyhydramnios was analysed (Table 2).

Regarding complications during childbirth and postpartum period, there was a relationship between BMI and intrapartum fever, preeclampsia, induction of childbirth, type of delivery, presence of episiotomy, and birth composite morbidity (variable formed by the presence of all the complications during childbirth). No relationship was found with deep vein thrombosis, oligohydramnios, polyhydramnios, uterine rupture, general anaesthesia, grade III and IV perineal tear, postpartum surgery, maternal admittance to ICU, and maternal re-admittance to hospital. As for the newborn, children born to obese mothers were more likely to suffer from macrosomia and to be admitted to neonates' units. Details of this analysis can be found in Table 3 .

Following this analysis (Table 4), we observed that skin-to-skin contact was performed in $77.9 \%$ of women (4574), the onset of breastfeeding after delivery in $74.5 \%$ (4372) of women, and 79.7\% (4681) breastfed their baby at hospital discharge. When performing both the bivariate and the multivariate analyses, the three study variables were related with BMI. Thus, we observed that obese women experienced a lower rate of skin-to-skin contact at birth with an AOR of 0.79 for obesity type I (95\% CI $0.65,0.97)$, of 0.68 for obesity type II $(95 \% \mathrm{CI}$ $0.50,0.93$ ), and of 0.51 for obesity type III ( $95 \%$ CI 0.32 , 0.83 ), as compared to mothers with normoweight. Lower rates of breastfeeding initiation were also observed in the first hour with an AOR of 0.80 for obesity type I (95\% CI $0.66,0.97)$, of 0.66 for obesity type II $(95 \%$ CI $0.49,0.90)$, and of 0.58 for obesity type III (95\% CI 0.36 , 0.94 ), as compared to mothers with normoweight. Likewise, a decrease in the probability of exclusive breastfeeding at discharge was observed for type III obese mothers, with an AOR of 0.57 (95\% CI 0.35, 0.94), as compared to mothers with normoweight. These associations coincided when the analysis was performed by introducing the BMI as a quantitative variable.

Women were then asked about the different complications and/or problems that could have arisen throughout hospitalisation and at home after discharge. After the multivariate analysis, a link between maternal obesity in childbirth and various complications was observed. However, only a linear trend was observed during hospital admission between obesity and reduced breast engorgement, with an AOR of 0.70 for obesity type I (of 
Table 1 Sociodemographic characteristics of the women according to their BMI

\begin{tabular}{|c|c|c|c|c|c|c|c|}
\hline \multirow[t]{2}{*}{ Variable } & \multicolumn{5}{|l|}{$\mathrm{BMI}$} & \multirow[b]{2}{*}{$\begin{array}{l}\text { Total Values } \\
n(\%)\end{array}$} & \multirow[b]{2}{*}{$P$ - value* } \\
\hline & $\begin{array}{l}\text { Normoweight } \\
n(\%)\end{array}$ & $\begin{array}{l}\text { Overweight } \\
n(\%)\end{array}$ & $\begin{array}{l}\text { Obesity type I } \\
n(\%)\end{array}$ & $\begin{array}{l}\text { Obesity type II } \\
n(\%)\end{array}$ & $\begin{array}{l}\text { Obesity type III } \\
\text { n (\%) }\end{array}$ & & \\
\hline Maternal age & & & & & & & $<0.001$ \\
\hline$<25$ years & $38(2.4)$ & $60(2.2)$ & $34(2.9)$ & $10(3.4)$ & $7(7.3)$ & $239(4)$ & $x^{2}=16.2$ \\
\hline $25-35$ & $938(58.4)$ & $1545(56.8)$ & $697(60.5)$ & $192(65.5)$ & $66(68.8)$ & $3438(57.6)$ & $d f=1$ \\
\hline$>35$ years & $631(39.3)$ & $1117(41.0)$ & $422(36.6)$ & $91(31.1)$ & $23(24.0)$ & $2284(38.3)$ & \\
\hline Level of education & & & & & & & $<0.001$ \\
\hline None & $0(0.0)$ & $4(0.1)$ & $2(0.2)$ & $2(0.7)$ & $0(0.0)$ & $8(0.1)$ & $x^{2}=99.9$ \\
\hline Primary & $28(1.7)$ & $48(1.8)$ & $21(1.8)$ & $6(2.0)$ & $3(3.1)$ & $106(1.8)$ & $d f=1$ \\
\hline Secondary & $340(21.2)$ & $715(26.3)$ & $379(32.9)$ & $132(45.1)$ & $45(46.9)$ & $1611(27.4)$ & \\
\hline University & $1239(77.1)$ & $1955(71.8)$ & $751(65.1)$ & $153(52.2)$ & $48(50.0)$ & $4146(70.6)$ & \\
\hline $\begin{array}{l}\text { Monthly family } \\
\text { income (euros) }\end{array}$ & & & & & & & $<0.001$ \\
\hline$<1000$ & $81(5.0)$ & $139(5.1)$ & $74(6.4)$ & $30(10.2)$ & $9(9.4)$ & $333(5.7)$ & $x^{2}=69.4$ \\
\hline $1000-2000$ & $469(29.2)$ & $891(32.7)$ & $416(36.1)$ & $128(43.7)$ & $45(46.9)$ & $1849(32)$ & $d f=1$ \\
\hline $2000-3000$ & $571(35.5)$ & $924(33.9)$ & $397(34.4)$ & $83(28.3)$ & $29(30.2)$ & 2004 (34.7) & \\
\hline $3000-4000$ & 331 (20.6) & $533(19.6)$ & $202(17.5)$ & $41(14.0)$ & $9(9.4)$ & $1116(19.3)$ & \\
\hline$>4000$ & 155 (9.6) & 235 (8.6) & $64(5.6)$ & $11(3.8)$ & $4(4.2)$ & $469(8.1)$ & \\
\hline Nationality & & & & & & & 0.91 \\
\hline Spanish & 1508 (93.8) & $2557(93.9)$ & $1076(93.3)$ & $276(94.2)$ & $92(95.8)$ & 5509 (93.8) & $x^{2}=0.01$ \\
\hline Foreign & $99(6.2)$ & $165(6.1)$ & $77(6.7)$ & $17(5.8)$ & $4(4.2)$ & $362(6.2)$ & $d f=1$ \\
\hline $\begin{array}{l}\text { Attendance to } \\
\text { maternal education }\end{array}$ & & & & & & & 0.36 \\
\hline No & $375(23.3)$ & $602(22.1)$ & $271(23.5)$ & $61(20.8)$ & 35 (36.5) & $1344(22.8)$ & $x^{2}=0.86$ \\
\hline Yes & $1232(76.7)$ & $2120(77.9)$ & $882(76.5)$ & $232(79.2)$ & $61(63.5)$ & $4527(77.2)$ & $d f=1$ \\
\hline $\begin{array}{l}\text { Pre-pregnancy } \\
\text { smoking habits }\end{array}$ & & & & & & & $<0.001$ \\
\hline No & 1296 (80.6) & 2104 (77.3) & $834(72.3)$ & 201 (68.6) & 69 (71.9) & $4504(76.7)$ & $x^{2}=36.1$ \\
\hline Yes & 311 (19.4) & $618(22.7)$ & $319(27.7)$ & $92(31.4)$ & $27(28.1)$ & $1367(23.3)$ & $d f=1$ \\
\hline
\end{tabular}

$0.70)(95 \%$ CI $0.55,0.89)$, of 0.63 for obesity type II $(95 \%$ CI $0.42,0.94$ ), and of 0.42 for obesity type III (95\% CI $0.19,0.94)$, as compared to women with normoweight, as well as increased feelings of nervousness with an AOR of 1.23 for overweight women (of 1.23) (95\% CI $1.06,1.43)$, of 1.37 for obesity type I (95\% CI 1.15, 1.64), of 1.60 for obesity type II $(95 \%$ CI $1.20,2.14)$, and of 1.69 for obesity type III $(95 \%$ CI 1.05, 2.74), as compared to women with normoweight.

Once at home, the mothers also had lower breast engorgement with an AOR of 0.58 for obesity type II (95\% CI $0.43,0.77)$, and of 0.33 for obesity type III (95\% CI $0.19,0.58)$, an increased rise of delayed breast milk with an AOR of 1.31 for obesity type I (95\% CI 1.01, 1.71), of 1.43 for obesity type II (95\% CI $0.94,2.16)$, and of 1.83 for obesity type III $(95 \%$ CI $0.96,3.50)$, as well as more problems related to the baby not gaining weight, with an AOR of 1.37 for obesity type II (95\% CI 1.01, 1.84), and of 1.79 for obesity type III (95\% CI 1.10, 2.90). When the analysis was again performed though including BMI as a quantitative variable, all the associations coincided except for difficulties with positioning the child due to C-section injury or episiotomy/tearing stitching, which showed a significant relationship. However, no relationship was found regarding baby not gaining weight. All the details of this analysis can be found in Table 5 .

\section{Discussion}

In this study we have investigated the relationship between BMI and the risk of complications during breastfeeding and its associated problems. Specifically, we have observed a linear relationship between higher BMI figures and a decrease in the likelihood of starting skin-to-skin contact, breastfeeding in the first hour, and exclusive breastfeeding at hospital discharge (Table 4). In addition, we also found a higher perception of 
Table 2 Obstetric characteristics of the women according to their BMI

\begin{tabular}{|c|c|c|c|c|c|c|c|}
\hline \multirow[t]{2}{*}{ Variable } & \multicolumn{5}{|l|}{$\mathrm{BMI}$} & \multirow[b]{2}{*}{$\begin{array}{l}\text { Total values } \\
n(\%)\end{array}$} & \multirow[b]{2}{*}{$P$ - value } \\
\hline & $\begin{array}{l}\text { Normoweight } \\
n(\%)\end{array}$ & $\begin{array}{l}\text { Overweight } \\
n(\%)\end{array}$ & $\begin{array}{l}\text { Obesity type I } \\
n(\%)\end{array}$ & $\begin{array}{l}\text { Obesity type II } \\
n(\%)\end{array}$ & $\begin{array}{l}\text { Obesity type III } \\
n(\%)\end{array}$ & & \\
\hline Number of pregnancies & & & & & & & 0.006 \\
\hline One & $860(53.5)$ & $1456(53.5)$ & $611(53.0)$ & $149(50.9)$ & $50(52.1)$ & $3126(56.1)$ & $x^{2}=7.4$ \\
\hline Two & $562(35.0)$ & $910(33.4)$ & $356(30.9)$ & $99(33.8)$ & $31(32.3)$ & $1658(29.7)$ & $d f=1$ \\
\hline Three & $137(8.5)$ & $232(8.5)$ & $120(10.4)$ & $33(11.3)$ & $11(11.5)$ & $533(9.5)$ & \\
\hline Four & $36(2.2)$ & $84(3.1)$ & $39(3.4)$ & $8(2.7)$ & $2(2.1)$ & 169 (3) & \\
\hline Five or more & $12(0.7)$ & $40(1.5)$ & $27(2.3)$ & $4(1.4)$ & $2(2.1)$ & $85(1.5)$ & \\
\hline Number of deliveries & & & & & & & $<0.001$ \\
\hline None & $259(16.1)$ & $595(21.9)$ & $311(27.0)$ & $98(33.4)$ & $33(34.4)$ & $1296(22.1)$ & $x^{2}=40.0$ \\
\hline One & $869(54.1)$ & $1391(51.1)$ & $542(47.0)$ & $132(45.1)$ & $40(41.7)$ & $2974(50.7)$ & $d f=1$ \\
\hline Two & $422(26.3)$ & $645(23.7)$ & $261(22.6)$ & $50(17.1)$ & $20(20.8)$ & $1398(23.8)$ & \\
\hline Three & $48(3.0)$ & $80(2.9)$ & $33(2.9)$ & $10(3.4)$ & $3(3.1)$ & $164(2.8)$ & \\
\hline Four & $9(0.6)$ & $10(0.4)$ & $4(0.3)$ & $1(0.3)$ & $0(0.0)$ & $24(0.4)$ & \\
\hline Five or more & $0(0.0)$ & $1(0.0)$ & $2(0.2)$ & $2(0.7)$ & $0(0.0)$ & $5(0.0)$ & \\
\hline Twin pregnancy & & & & & & & 0.004 \\
\hline No & $1583(98.5)$ & $2652(97.4)$ & $1118(97.0)$ & $284(96.9)$ & $92(95.8)$ & $5729(97.6)$ & $x^{2}=8.5$ \\
\hline Yes & $24(1.5)$ & $70(2.6)$ & $35(3.0)$ & $9(3.1)$ & $4(4.2)$ & $142(2.4)$ & $d f=1$ \\
\hline Prematurity & & & & & & & 0.03 \\
\hline$>37$ & $1472(91.6)$ & $2557(93.9)$ & $1065(92.4)$ & $278(94.9)$ & $93(96.9)$ & $5465(93.1)$ & $x^{2}=4.5$ \\
\hline$<37$ & $135(8.4)$ & $165(6.1)$ & $88(7.6)$ & $15(5.1)$ & $3(3.1)$ & $406(6.9)$ & $d f=1$ \\
\hline Hypertensive states & & & & & & & $<0.001$ \\
\hline No & $1550(96.5)$ & $2568(94.3)$ & $1032(89.5)$ & $239(81.6)$ & $77(80.2)$ & $5466(93.1)$ & $x^{2}=130.7$ \\
\hline Yes & $57(3.5)$ & $154(5.7)$ & $121(10.5)$ & $54(18.4)$ & $19(19.8)$ & $405(6.9)$ & $d f=1$ \\
\hline Gestational diabetes-diet & & & & & & & $<0.001$ \\
\hline No & $1429(88.9)$ & $2463(90.5)$ & $988(85.7)$ & $223(76.1)$ & $75(78.1)$ & $5178(88.2)$ & $x^{2}=39.7$ \\
\hline Yes & $178(11.1)$ & $259(9.5)$ & $165(14.3)$ & $70(23.9)$ & $21(21.9)$ & $693(11.8)$ & $d f=1$ \\
\hline Gestational diabetes-insulin & & & & & & & $<0.001$ \\
\hline No & $1575(98.0)$ & $2660(97.7)$ & $1124(97.5)$ & $275(93.9)$ & $89(92.7)$ & $5723(97.5)$ & $x^{2}=16.1$ \\
\hline Yes & $32(2.0)$ & $62(2.3)$ & $29(2.5)$ & $18(6.1)$ & $7(7.3)$ & $148(2.5)$ & $d f=1$ \\
\hline Hyperthyroidism & & & & & & & 0.34 \\
\hline No & $1528(95.1)$ & $2601(95.6)$ & $1100(95.4)$ & $272(92.8)$ & $90(93.8)$ & $5591(95.2)$ & $x^{2}=0.9$ \\
\hline Yes & $79(4.9)$ & $121(4.4)$ & $53(4.6)$ & $21(7.2)$ & $6(6.3)$ & $280(4.7)$ & $d f=1$ \\
\hline Hypothyroidism & & & & & & & 0.43 \\
\hline No & $1398(87.0)$ & $2375(87.3)$ & $984(85.3)$ & $251(85.7)$ & $86(89.6)$ & $5094(86.7)$ & $x^{2}=0.6$ \\
\hline Yes & $209(13.0)$ & $347(12.7)$ & $169(14.7)$ & $42(14.3)$ & $10(10.4)$ & 777 (13.3) & $d f=1$ \\
\hline Anaemia & & & & & & & 0.03 \\
\hline No & $967(60.2)$ & $1605(59.0)$ & $732(63.5)$ & $186(63.5)$ & $63(65.6)$ & $3553(60.5)$ & $x^{2}=4.5$ \\
\hline Yes & $640(39.8)$ & $1117(41.0)$ & $421(36.5)$ & $107(36.5)$ & $33(34.4)$ & 2318 (39.5) & $d f=1$ \\
\hline & & & & & & & 0.50 \\
\hline No & $1584(98.6)$ & $2691(98.9)$ & 1137 (98.6) & $291(99.3)$ & $95(99.0)$ & $5798(98.7)$ & $x^{2}=0.5$ \\
\hline Yes & $23(1.4)$ & $31(1.1)$ & $16(1.4)$ & $2(0.7)$ & $1(1.0)$ & $73(1.3)$ & $d f=1$ \\
\hline Risk of preterm birth & & & & & & & 0.003 \\
\hline No & $1433(89.2)$ & $2529(92.9)$ & $1062(92.1)$ & $276(94.2)$ & $87(90.6)$ & $5387(91.7)$ & $x^{2}=8.9$ \\
\hline Yes & $174(10.8)$ & $193(7.1)$ & $91(7.9)$ & $17(5.8)$ & $9(9.4)$ & $484(8.2)$ & $d f=1$ \\
\hline
\end{tabular}


Table 2 Obstetric characteristics of the women according to their BMI (Continued)

\begin{tabular}{|c|c|c|c|c|c|c|c|}
\hline \multirow[t]{2}{*}{ Variable } & \multicolumn{5}{|l|}{$\mathrm{BMI}$} & \multirow[b]{2}{*}{$\begin{array}{l}\text { Total values } \\
n(\%)\end{array}$} & \multirow[b]{2}{*}{$P$ - value* } \\
\hline & $\begin{array}{l}\text { Normoweight } \\
n(\%)\end{array}$ & $\begin{array}{l}\text { Overweight } \\
n(\%)\end{array}$ & $\begin{array}{l}\text { Obesity type I } \\
n(\%)\end{array}$ & $\begin{array}{l}\text { Obesity type II } \\
n(\%)\end{array}$ & $\begin{array}{l}\text { Obesity type III } \\
n(\%)\end{array}$ & & \\
\hline Deep vein thrombosis & & & & & & & 0.75 \\
\hline No & $1580(98.3)$ & $2695(99.0)$ & $1138(98.7)$ & $289(98.6)$ & $92(95.8)$ & $5794(98.6)$ & $x^{2}=0.1$ \\
\hline Yes & $27(1.7)$ & $27(1.0)$ & $15(1.3)$ & $4(1.4)$ & $4(4.2)$ & $77(1.3)$ & $d f=1$ \\
\hline Oligohydramnios & & & & & & & 0.07 \\
\hline No & $1561(97.1)$ & $2618(96.2)$ & $1092(94.7)$ & $285(97.3)$ & $93(96.9)$ & $5649(96.2)$ & $x^{2}=3.3$ \\
\hline Yes & $46(2.9)$ & $104(3.8)$ & $61(5.3)$ & $8(2.7)$ & $3(3.1)$ & $222(3.8)$ & $d f=1$ \\
\hline Polyhydramnios & & & & & & & 0.001 \\
\hline No & $1502(93.5)$ & $2480(91.1)$ & $1037(89.9)$ & $259(88.4)$ & $82(85.4)$ & $5360(91.3)$ & $x^{2}=18.8$ \\
\hline Yes & $105(6.5)$ & $242(8.9)$ & $116(10.1)$ & $34(11.6)$ & $14(14.6)$ & $511(8.7)$ & $d f=1$ \\
\hline $\begin{array}{l}\text { Composite pregnancy } \\
\text { morbidity }\end{array}$ & & & & & & & 0.02 \\
\hline No & $621(38.6)$ & 1051 (38.6) & 419 (36.3) & $93(31.7)$ & $31(32.3)$ & $2215(37.7)$ & $x^{2}=5.9$ \\
\hline Yes & $986(61.4)$ & $1671(61.4)$ & 734 (63.7) & $200(68.3)$ & 65 (67.7) & 3656 (62.3) & $d f=1$ \\
\hline
\end{tabular}

*Pearson's $x^{2}$ test. df: degrees of freedom. Statistical significance at $p \leq 0.05$

lactogenesis delay and low weight gain in the newborn among the most important results (Table 5), coinciding with other authors $[9,12]$ that related BMI with late lactogenesis.

On the other hand, while this was not the study objective, we found what is in line with other research $[6,13,16]$, that is an association between obesity and the risk of certain complications during pregnancy such as prematurity, hypertensive disorders in pregnancy, gestational diabetes (both controlled with insulin and through diet), risk of premature birth, deep vein thrombosis, oligohydramnios and polyhydramnios. Regarding complications during childbirth and postpartum, an increased risk of intrapartum fever [17], preeclampsia [6, 18], induction of delivery and caesarean delivery [6] was also observed. As for the newborn, children born to obese mothers were more likely suffer from macrosomy [6] and to be admitted to neonatal units $[3,19]$.

In this sense, our results coincide with those from other studies in which not only a lower likelihood of starting breastfeeding is observed [9-11], but also shorter duration if commenced [8]. Among the causes that may explain the lower probability of breastfeeding in obese women we find, on one hand, those related to all obstetric complications associated with obesity and, on the other, those related to differences attributable to a worse neuroendocrine response during the lactogenesis process. At the same time, other aspects of the psychosocial sphere [20] associated with obesity such as distress [10], increased risk of depression [10, 21], discrimination [22], worse body image [23, 24], social stigma [22], etc. may also influence.

Among the variables related to obstetric complications, the type of delivery stands out (Table 3). Obese women have an increased risk of C-sections [6, 25] and this is, in turn, associated with a greater premature separation of the mother-child binomial, thus hindering skin-to-skin contact and the onset of breastfeeding. However, we cannot make comparisons as no studies have been found that have delved into these aspects.

As for the neuroendocrine differences with respect to women with normoweight, there are several factors that can contribute to a delay in lactogenesis. First, it is known that decreased progesterone levels, which occurs after delivery, is one of the responsible factors for the preparation of the mammary gland [26]. Because progesterone is stored in adipose tissue, obese women may have more consistent hormone levels that may be responsible for inhibiting lactogenesis [4]. Second, another factor that could indirectly influence the perceived delay in lactogenesis is the anatomical characteristics of the obese woman's breast, as adipose tissue between the ducts could affect the proper flow of milk [4]. Finally, there are certain disorders such as diabetes that, associated with maternal obesity, could interfere with the breastfeeding process. Women with diabetes and those who have a C-section may be more likely to experience delayed or low milk levels of lactogenesis [19]. These results could be explained by the lower prolactin concentration shown in obese mothers at rest and after the breastfeeding episode $[4,27]$. Based on this fact and on our results, there would be several possible lines of action. On the one hand, as primary prevention measure, women should be recommended to reduce their BMI in pre-conception consultations so as to, among other benefits, attain successful breastfeeding. On the other hand, during breastfeeding, obese women should be encouraged to feed their children more often and that 
Table 3 Delivery and postpartum complications according to maternal BMI

\begin{tabular}{|c|c|c|c|c|c|c|c|}
\hline \multirow[t]{2}{*}{ Variable } & \multicolumn{5}{|l|}{$\mathrm{BMI}$} & \multirow[b]{2}{*}{$\begin{array}{l}\text { Total values } \\
\mathrm{n}(\%)\end{array}$} & \multirow[b]{2}{*}{$P$ - value* } \\
\hline & $\begin{array}{l}\text { Normoweight } \\
N(\%)\end{array}$ & $\begin{array}{l}\text { Overweight } \\
\mathrm{n}(\%)\end{array}$ & $\begin{array}{l}\text { Obesity type I } \\
\mathrm{n}(\%)\end{array}$ & $\begin{array}{l}\text { Obesity type ॥ } \\
\mathrm{n}(\%)\end{array}$ & $\begin{array}{l}\text { Obesity type III } \\
\text { n (\%) }\end{array}$ & & \\
\hline Uterine rupture & & & & & & & 0.54 \\
\hline No & $1595(99.3)$ & $2708(99.5)$ & $1148(99.6)$ & $288(98.3)$ & $95(99.0)$ & $5834(99.3)$ & $x^{2}=0.8$ \\
\hline Yes & $12(0.7)$ & $14(0.5)$ & $5(0.4)$ & $5(1.7)$ & $1(1.0)$ & $37(0.7)$ & $d f=1$ \\
\hline Fever & & & & & & & $<0.001$ \\
\hline No & $1540(95.8)$ & $2574(94.6)$ & $1081(93.8)$ & $275(93.9)$ & $84(87.5)$ & 5554 (94.6) & $x^{2}=12.1$ \\
\hline Yes & $67(4.2)$ & $148(5.4)$ & $72(6.2)$ & $18(6.1)$ & $12(12.5)$ & $317(5.4)$ & $d f=1$ \\
\hline Preeclampsia & & & & & & & $<0.001$ \\
\hline No & $1577(98.1)$ & $2654(97.5)$ & $1103(95.7)$ & $266(90.8)$ & $85(88.5)$ & $5685(96.8)$ & $x^{2}=60.0$ \\
\hline Yes & $30(1.9)$ & $68(2.5)$ & $50(4.3)$ & $27(9.2)$ & $11(11.5)$ & $186(3.2)$ & $d f=1$ \\
\hline Epidural analgesia & & & & & & & $<0.001$ \\
\hline No & $524(32.6)$ & $802(29.5)$ & $314(27.2)$ & $66(22.5)$ & $21(21.9)$ & $1727(29.4)$ & $x^{2}=19.4$ \\
\hline Yes & $1083(67.4)$ & $1920(70.5)$ & $839(72.8)$ & $227(77.5)$ & $75(78.1)$ & $4144(70.6)$ & $d f=1$ \\
\hline General anaesthesia & & & & & & & 0.09 \\
\hline No & $1590(98.9)$ & $2692(98.9)$ & 1137 (98.6) & $285(97.3)$ & $95(97.3)$ & $5799(98.8)$ & $x^{2}=2.86$ \\
\hline Yes & $17(1.1)$ & $30(1.1)$ & $16(1.4)$ & $8(2.7)$ & $1(1.0)$ & $72(1.2)$ & $d f=1$ \\
\hline Induced delivery & & & & & & & $<0.001$ \\
\hline No & $1121(69.8)$ & $1708(62.7)$ & $688(59.7)$ & $151(51.5)$ & $52(54.2)$ & $3720(63.3)$ & $x^{2}=52.5$ \\
\hline Yes & $486(30.2)$ & $1014(37.3)$ & $465(40.3)$ & $142(48.5)$ & $44(45.8)$ & $2151(36.7)$ & $d f=1$ \\
\hline Mode of delivery & & & & & & & $<0.001$ \\
\hline Vaginal & $1075(66.9)$ & $1629(59.8)$ & $639(55.4)$ & $136(46.4)$ & $47(49.0)$ & $3526(60.0)$ & $x^{2}=101.2$ \\
\hline Instrumental & $264(16.4)$ & $449(16.5)$ & $176(15.3)$ & $46(15.7)$ & $14(14.6)$ & $949(16.1)$ & $d f=1$ \\
\hline Planned caesarean & $89(5.5)$ & $220(8.1)$ & $108(9.4)$ & $41(14.0)$ & $6(6.3)$ & $464(7.9)$ & \\
\hline Emergency caesarean & $179(11.1)$ & $424(15.6)$ & $230(19.9)$ & $70(23.9)$ & $29(30.2)$ & 935 (15.9) & \\
\hline Episiotomy & & & & & & & $<0.001$ \\
\hline No & $1030(64.1)$ & $1812(66.6)$ & $822(71.3)$ & $218(74.4)$ & $74(77.1)$ & $3956(67.4)$ & $x^{2}=26.6$ \\
\hline Yes & $577(35.9)$ & $910(33.4)$ & $331(28.7)$ & $75(25.6)$ & $22(22.9)$ & 1915 (32.6) & $d f=1$ \\
\hline III-IV tearing & & & & & & & 0.07 \\
\hline No & $1589(98.9)$ & $2689(98.8)$ & $1136(98.5)$ & $284(96.9)$ & $95(99.0)$ & $5793(98.7)$ & $x^{2}=3.3$ \\
\hline Yes & 181.1 & $33(1.2)$ & $17(1.5)$ & $9(3.1)$ & $1(1.0)$ & $78(1.3)$ & $d f=1$ \\
\hline $\begin{array}{l}\text { Composite morbidity } \\
\text { delivery }\end{array}$ & & & & & & & $<0.001$ \\
\hline No & $728(45.3)$ & 995 (36.6) & $336(29.1)$ & $57(19.5)$ & $18(18.8)$ & $21.34(36.7)$ & $x^{2}=128.6$ \\
\hline Yes & $879(54.7)$ & $1727(63.4)$ & $817(70.9)$ & $236(80.5)$ & $78(81.3)$ & $3737(63.3)$ & $d f=1$ \\
\hline $\begin{array}{l}\text { Postpartum surgery } \\
\text { related to delivery }\end{array}$ & & & & & & & 0.10 \\
\hline No & $1551(96.5)$ & $2634(96.8)$ & $1105(95.8)$ & $280(95.6)$ & $90(93.8)$ & $5660(96.4)$ & $x^{2}=2.6$ \\
\hline Yes & $56(3.5)$ & $88(3.2)$ & $48(4.2)$ & $13(4.4)$ & $6(6.3)$ & $211(3.6)$ & $d f=1$ \\
\hline $\begin{array}{l}\text { Postpartum maternal } \\
\text { ICU admission }\end{array}$ & & & & & & & 0.43 \\
\hline No & $1575(98.0)$ & $2681(98.5)$ & $1128(97.8)$ & $285(97.3)$ & $94(97.9)$ & $5763(98.2)$ & $x^{2}=0.6$ \\
\hline Yes & $32(2.0)$ & $41(1.5)$ & $25(2.2)$ & $8(2.7)$ & $2(2.1)$ & $108(1.83)$ & $d f=1$ \\
\hline $\begin{array}{l}\text { Readmission after } \\
\text { discharge }\end{array}$ & & & & & & & 0.11 \\
\hline No & $1580(98.3)$ & $2666(97.9)$ & $1132(98.2)$ & $281(95.9)$ & $94(97.9)$ & $5753(98.0)$ & $x^{2}=2.6$ \\
\hline Yes & $27(1.7)$ & $56(2.1)$ & $21(1.8)$ & $12(4.1)$ & $2(2.1)$ & $118(2.0)$ & $d f=1$ \\
\hline Composite postpartum & & & & & & & 0.08 \\
\hline
\end{tabular}


Table 3 Delivery and postpartum complications according to maternal BMI (Continued)

\begin{tabular}{|c|c|c|c|c|c|c|c|}
\hline \multirow[t]{2}{*}{ Variable } & \multicolumn{5}{|l|}{ BMI } & \multirow[b]{2}{*}{$\begin{array}{l}\text { Total values } \\
\mathrm{n}(\%)\end{array}$} & \multirow[b]{2}{*}{$P$ - value* } \\
\hline & $\begin{array}{l}\text { Normoweight } \\
\text { N (\%) }\end{array}$ & $\begin{array}{l}\text { Overweight } \\
\mathrm{n}(\%)\end{array}$ & $\begin{array}{l}\text { Obesity type I } \\
\text { n (\%) }\end{array}$ & $\begin{array}{l}\text { Obesity type II } \\
\text { n (\%) }\end{array}$ & $\begin{array}{l}\text { Obesity type III } \\
\text { n (\%) }\end{array}$ & & \\
\hline \multicolumn{8}{|l|}{ morbidity } \\
\hline No & $1507(93.8)$ & $2558(94.0)$ & $1071(92.9)$ & $265(90.4)$ & $86(89.6)$ & $5487(93.5)$ & $x^{2}=5.6$ \\
\hline Yes & $100(6.2)$ & $164(6.0)$ & $82(7.1)$ & $28(9.6)$ & $10(10.4)$ & $384(6.5)$ & $d f=1$ \\
\hline Low birthweight & & & & & & & 0.13 \\
\hline No & $1488(92.7)$ & $2560(94.3)$ & $1076(93.4)$ & $271(93.1)$ & $95(99.0)$ & $5490(93.5)$ & $x^{2}=2.3$ \\
\hline Yes & $118(7.3)$ & $155(5.7)$ & $76(6.6)$ & $20(6.9)$ & $1(1.0)$ & $370(6.5)$ & $d f=1$ \\
\hline Macrosomia & & & & & & & $<0.001$ \\
\hline No & $1561(97.2)$ & $2608(96.1)$ & $1072(93.1)$ & $267(91.8)$ & $81(84.4)$ & $5589(95.2)$ & $x^{2}=56.4$ \\
\hline Yes & $45(2.8)$ & $107(3.9)$ & $80(6.9)$ & $24(8.2)$ & 15 (15.6) & $271(4.8)$ & $\mathrm{df}=1$ \\
\hline Newborn admission & & & & & & & $<0.001$ \\
\hline No & $1422(88.5)$ & $2399(88.1)$ & $991(85.9)$ & $231(78.8)$ & $82(85.4)$ & $5125(87.3)$ & $x^{2}=15.2$ \\
\hline Yes & 185 (11.5) & $323(11.9)$ & $162(14.1)$ & $62(21.2)$ & 14 (14.6) & $746(12.7)$ & $d f=1$ \\
\hline
\end{tabular}

*Pearson's $\mathrm{X}^{2}$ test. df: degrees of freedom. Statistical significance at $p \leq 0.05$

Table 4 Breastfeeding complications according to the BMI

\begin{tabular}{|c|c|c|c|c|c|c|}
\hline \multirow[t]{2}{*}{ Variable } & \multicolumn{5}{|l|}{$\mathrm{BMI}$} & \multirow[b]{2}{*}{$\begin{array}{l}\text { Total values } \\
\boldsymbol{n}(\%)\end{array}$} \\
\hline & $\begin{array}{l}\text { Normoweight } \\
\boldsymbol{n}(\%)\end{array}$ & $\begin{array}{l}\text { Overweight } \\
\boldsymbol{n}(\%)\end{array}$ & $\begin{array}{l}\text { Obesity type I } \\
\boldsymbol{n}(\%)\end{array}$ & $\begin{array}{l}\text { Obesity type II } \\
\boldsymbol{n}(\%)\end{array}$ & $\begin{array}{l}\text { Obesity type III } \\
\boldsymbol{n}(\%)\end{array}$ & \\
\hline \multicolumn{7}{|l|}{ Skin-to-skin contact } \\
\hline No & 300 (18.7) & $562(20.6)$ & $304(26.4)$ & $96(32.8)$ & 35 (36.5) & 1297(22.1) \\
\hline Yes & $1307(81.3)$ & $2160(79.4)$ & 849 (73.6) & $197(67.2)$ & $61(63.5)$ & $4574(77.9)$ \\
\hline OR Cl 95 & 1 (ref.) & $0.88(0.76,1.03)$ & $0.64(0.54,0.77)$ & $0.47(0.36,0.62)$ & $0.40(0.26,0.62)$ & \\
\hline${ }^{\mathrm{a}} \mathrm{AOR}$ Cl 95\% & 1 (ref.) & $0.97(0.82,1.16)$ & $0.79(0.65,0.97)$ & $0.68(0.50,0.93)$ & $0.51(0.32,0.83)$ & \\
\hline aAOR CI 95\% Total & $0.96(0.95,0.98)$ & & & & & \\
\hline \multicolumn{7}{|l|}{$\begin{array}{l}\text { Start of breastfeeding } \\
\text { in the first hour }\end{array}$} \\
\hline No & $358(22.3)$ & $661(24.3)$ & $339(29.4)$ & $106(36.2)$ & $35(36.5)$ & 1499 (25.5) \\
\hline Yes & $1249(77.7)$ & $2061(75.7)$ & $814(70.6)$ & $187(63.8)$ & $61(63.5)$ & $4372(74.5)$ \\
\hline OR CI 95\% & 1 (ref.) & $0.89(0.77,1.04)$ & $0.69(0.58,0.82)$ & $0.51(0.39,0.66)$ & $0.50(0.32,0.77)$ & \\
\hline${ }^{\mathrm{b}} \mathrm{AOR}$ CI $95 \%$ & 1 (ref.) & $0.93(0.79,1.10)$ & $0.80(0.66,0.97)$ & $0.66(0.49,0.90)$ & $0.58(0.36,0.94)$ & \\
\hline${ }^{\mathrm{b}} \mathrm{AOR} \mathrm{Cl}$ 95\% Total & $0.97(0.95,0.98)$ & & & & & \\
\hline \multicolumn{7}{|c|}{$\begin{array}{l}\text { Exclusive breastfeeding } \\
\text { at discharge }\end{array}$} \\
\hline No & $286(17.8)$ & $530(19.5)$ & $272(23.6)$ & $74(25.3)$ & $28(29.2)$ & $1190(20.3)$ \\
\hline Yes & $1321(82.2)$ & $2192(80.5)$ & $881(76.4)$ & $219(74.7)$ & $68(70.8)$ & 4681 (79.7) \\
\hline OR CI 95\% & 1 (ref.) & $0.90(0.76,1.05)$ & $0.70(0.58,0.85)$ & $0.64(0.48,0.86)$ & $0.53(0.33,0.83)$ & \\
\hline${ }^{\mathrm{b}} \mathrm{AOR} \mathrm{Cl} 95 \%$ & 1 (ref.) & $0.91(0.77,1.08)$ & $0.79(0.64,0.97)$ & $0.78(0.57,1.07)$ & $0.57(0.35,0.94)$ & \\
\hline${ }^{\mathrm{b}} \mathrm{AOR}$ Cl 95\% Total & $0.96(0.95-0.98)$ & & & & & \\
\hline
\end{tabular}

For the multivariate analysis, the ENTER method for binary logistic regression was used

All variables adjusted by: maternal age, nulliparity, twin pregnancy, previous caesarean, smoking habit, economic income, level of education, nationality, maternity training, pregnancy composite morbidity, delivery composite morbidity, postpartum composite morbidity and epidural analgesia

adjusted by: + prematurity

${ }^{\mathrm{b}}$ Adjusted by: + newborn admission + prematurity + breastfeeding previous children

Pregnancy composite morbidity: including preeclampsia during pregnancy, diet-controlled gestational diabetes, insulin-controlled gestational diabetes, hyperthyroidism, hypothyroidism, anaemia, intrahepatic cholestasis, risk of preterm birth, deep vein thrombosis

Delivery composite morbidity: including altered foetal heart rate (FHR) during delivery, stained amniotic fluid (AF), vaginal bleeding, non-progression of delivery, cephalopelvic disproportion, intrapartum fever (body temperature higher than $38^{\circ} \mathrm{C}$ ), intrapartum preeclampsia, induced delivery, end of delivery by caesarean, and severe tearing (type III-IV)

Postpartum composite morbidity: including maternal postpartum surgery related to the delivery, maternal admission to intensive care unit, and maternal re-admission 
Table 5 Complications and discomfort during hospitalisation and at home related to breastfeeding according to maternal BMI

\begin{tabular}{|c|c|c|c|c|c|c|}
\hline Variable & $\mathrm{BMI}$ & & & & & \\
\hline $\begin{array}{l}\text { Did you encounter any difficulty } \\
\text { with breastfeeding while in } \\
\text { hospital due to }\end{array}$ & $\begin{array}{l}\text { Normoweight } \\
\boldsymbol{n}(\%)\end{array}$ & $\begin{array}{l}\text { Overweight } \\
\boldsymbol{n}(\%)\end{array}$ & $\begin{array}{l}\text { Obesity type I } \\
\boldsymbol{n}(\%)\end{array}$ & $\begin{array}{l}\text { Obesity type II } \\
\boldsymbol{n}(\%)\end{array}$ & $\begin{array}{l}\text { Obesity type III } \\
\boldsymbol{n}(\%)\end{array}$ & $\begin{array}{l}\text { Total values } \\
\boldsymbol{n}(\%)\end{array}$ \\
\hline \multicolumn{7}{|l|}{ Pain? } \\
\hline No & $902(58.2)$ & $1508(57.8)$ & $616(55.9)$ & $154(55.6)$ & $50(58.8)$ & $3230(55.0)$ \\
\hline Yes & $647(41.8)$ & $1100(42.2)$ & $486(44.1)$ & $123(44.4)$ & $35(41.2)$ & $2391(45.0)$ \\
\hline OR Cl 95 & 1 (ref.) & $1.02(0.90,1.16)$ & $1.10(0.94,1.29)$ & $1.11(0.86,1.44)$ & $0.98(0.63,1.52)$ & \\
\hline aAOR CI 95\% & 1 (ref.) & $1.01(0.88,1.15)$ & $1.06(0.90,1.25)$ & $1.03(0.79,1.35)$ & $0.95(0.60,1.49)$ & \\
\hline${ }^{\mathrm{a}} \mathrm{AOR}$ Cl 95\% Total & $1.00(0.99,1.01)$ & & & & & \\
\hline \multicolumn{7}{|l|}{ Cracked nipples? } \\
\hline No & $1092(70.5)$ & $1777(68.1)$ & $754(68.5)$ & $189(68.2)$ & $64(74.4)$ & $3876(66.0)$ \\
\hline Yes & $457(29.5)$ & 831 (31.9) & $346(31.5)$ & $88(31.8)$ & $22(25.6)$ & $1744(34.0)$ \\
\hline OR CI 95\% & 1 (ref.) & $1.12(0.98,1.28)$ & $1.10(0.94,1.29)$ & $1.11(0.85,1.47)$ & $0.82(0.50,1.35)$ & \\
\hline${ }^{b} \mathrm{AOR} \mathrm{Cl} 95 \%$ & 1 (ref.) & $1.11(0.96,1.27)$ & $1.06(0.89,1.26)$ & $1.05(0.79,1.39)$ & $0.80(0.48-1.32)$ & \\
\hline${ }^{\mathrm{b}} \mathrm{AOR}$ Cl 95\% Total & $1.00(0.99,1.01)$ & & & & & \\
\hline \multicolumn{7}{|c|}{$\begin{array}{l}\text { Difficulties for latching on to } \\
\text { the breast/not proper latching on? }\end{array}$} \\
\hline No & $912(59.2)$ & $1481(56.7)$ & $575(52.1)$ & $134(48.6)$ & $51(60.0)$ & $3153(53.7)$ \\
\hline Yes & $629(40.8)$ & $1131(43.3)$ & $528(47.9)$ & $142(51.4)$ & $34(40.0)$ & $2464(46.3)$ \\
\hline OR CI 95\% & 1 (ref.) & $1.11(0.98,1.26)$ & $1.33(1.14,1.56)$ & $1.54(1.19,1.99)$ & $0.97(0.62,1.51)$ & \\
\hline${ }^{\mathrm{a}} \mathrm{AOR} \mathrm{Cl} 95 \%$ & 1 (ref.) & $1.11(0.97,1.27)$ & $1.27(1.08,1.50)$ & $1.42(1.08,1.87)$ & $0.86(0.54,1.37)$ & \\
\hline${ }^{\mathrm{a} A O R ~ C I} 95 \%$ Total & $1.02(1.00,1.03$ & & & & & \\
\hline \multicolumn{7}{|l|}{$\begin{array}{l}\text { Difficulties with positioning the } \\
\text { child due to C-section injury or } \\
\text { episiotomy/tearing stitching? }\end{array}$} \\
\hline No & $1290(83.5)$ & $2059(79.0)$ & $838(76.0)$ & $200(73.0)$ & $63(73.3)$ & $4450(75.8)$ \\
\hline Yes & $254(16.5)$ & $547(21.0)$ & $265(24.0)$ & $74(27.0)$ & $23(26.7)$ & $1163(24.2)$ \\
\hline OR CI 95\% & 1 (ref.) & $1.35(1.15,1.59)$ & $1.61(1.32,1.95)$ & $1.88(1.39,2.53)$ & $1.85(1.13,3.05)$ & \\
\hline${ }^{\mathrm{a}} \mathrm{AOR} \mathrm{Cl} 95 \%$ & 1 (ref.) & $1.22(1.03,1.46)$ & $1.29(1.05,1.58)$ & $1.32(0.96,1.82)$ & $1.27(0.75,2.16)$ & \\
\hline${ }^{\mathrm{a} A O R} \mathrm{Cl}$ 95\% Total & $1.02(1.00,1.04$ & & & & & \\
\hline \multicolumn{7}{|l|}{$\begin{array}{l}\text { Engorgement (swollen, tight, } \\
\text { hard, hot breasts...)? }\end{array}$} \\
\hline No & $1312(85.3)$ & $2227(85.7)$ & 969 (88.3) & $243(88.7)$ & 79 (91.9) & $4830(82.3)$ \\
\hline Yes & $227(14.7)$ & $373(14.3)$ & $129(11.7)$ & $31(11.3)$ & $7(8.1)$ & $767(17.7)$ \\
\hline OR CI 95\% & 1 (ref.) & $0.97(0.81,1.16)$ & $0.77(0.61,0.97)$ & $0.74(0.50,1.10)$ & $0.51(0.23,1.12)$ & \\
\hline${ }^{\mathrm{a}} \mathrm{AOR} \mathrm{Cl} 95 \%$ & 1 (ref.) & $0.94(0.78,1.13)$ & $0.70(0.55,0.89)$ & $0.63(0.42,0.94)$ & $0.42(0.19,0.94)$ & \\
\hline${ }^{\mathrm{a} A O R} \mathrm{Cl}$ 95\% Total & $0.96(0.94,0.97$ & & & & & \\
\hline \multicolumn{7}{|l|}{ Your own nervousness? } \\
\hline No & $1125(72.9)$ & $1790(68.7)$ & $713(64.8)$ & $165(60.2)$ & $51(60.0)$ & $3844(68.6)$ \\
\hline Yes & $418(27.1)$ & $814(31.3)$ & $387(35.2)$ & $109(39.8)$ & $34(40.0)$ & $1762(31.4)$ \\
\hline OR CI 95\% & 1 (ref.) & $1.22(1.07,1.41)$ & $1.46(1.24,1.73)$ & $1.78(1.36,2.32)$ & $1.79(1.15,2.81)$ & \\
\hline${ }^{\mathrm{a}} \mathrm{AOR} \mathrm{Cl} 95 \%$ & 1 (ref.) & $1.23(1.06,1.43)$ & $1.37(1.15-1.64)$ & $1.60(1.20,2.14)$ & $1.69(1.05,2.74)$ & \\
\hline${ }^{\mathrm{a} A O R ~ C I} 95 \%$ Total & $1.03(1.02,1.05$ & & & & & \\
\hline \multicolumn{7}{|l|}{ Your feeling unsure of yourself? } \\
\hline No & $987(64.0)$ & $1603(61.5)$ & $624(56.6)$ & $152(55.3)$ & $53(62.4)$ & $3419(60.9)$ \\
\hline Yes & $556(36.0)$ & $1002(38.5)$ & $478(43.4)$ & $123(44.7)$ & $32(37.6)$ & $2191(29.1)$ \\
\hline
\end{tabular}


Table 5 Complications and discomfort during hospitalisation and at home related to breastfeeding according to maternal BMI (Continued)

OR CI 95\%
${ }^{\mathrm{a} A O R ~ C I ~ 95 \% ~}$
${ }^{\mathrm{a}} \mathrm{AOR}$ Cl 95\% Total
Did you encounter any difficulty
with breastfeeding while at home due to

Pain?

No
Yes
OR CI 95\%
${ }^{b} A O R$ Cl 95\%
${ }^{b} A O R$ CI 95\% Total

\section{Cracked nipples?}

No
Yes
OR Cl 95\%
${ }^{\mathrm{b}} \mathrm{AOR}$ Cl 95\%
${ }^{\mathrm{b}} \mathrm{AOR}$ Cl 95\% Total

Difficulties for latching on to the breast/not proper latching on?

No
Yes
OR CI 95\%
${ }^{\mathrm{b}} \mathrm{AOR} \mathrm{Cl} \mathrm{95 \%}$
${ }^{\mathrm{b}} \mathrm{AOR} \mathrm{CI} 95 \%$ Total
Difficulties with positioning the
child due to C-section injury or
episiotomy/tearing stitching?

No
Yes
OR CI 95\%
${ }^{b}$ AOR CI 95\%
${ }^{b}$ AOR CI 95\% Total

\section{Engorgement (swollen, tight,} hard, hot breasts...)?

No
Yes
OR CI 95\%
${ }^{\mathrm{b}} \mathrm{AOR}$ Cl 95\%
${ }^{\mathrm{b}} \mathrm{AOR}$ CI 95\% Total

\section{Candidiasis (fungus)?}

\begin{tabular}{|c|}
\hline No \\
\hline Yes \\
\hline OR CI 95\% \\
\hline${ }^{\mathrm{b}} \mathrm{AOR}$ Cl 95\% \\
\hline${ }^{\mathrm{b}} \mathrm{AOR}$ CI 95\% Total \\
\hline
\end{tabular}

$\begin{array}{lrrrr}1 \text { (ref.) } & 1.11(0.97,1.26) & \mathbf{1 . 3 6}(\mathbf{1 . 1 6}, \mathbf{1 . 5 9}) & \mathbf{1 . 4 4}(\mathbf{1 . 1 1} \mathbf{1 . 8 6}) & 1.07(0.68,1.68) \\ 1 \text { (ref.) } & 1.15(0.99,1.32) & \mathbf{1 . 3 6}(\mathbf{1 . 1 5 ,} \mathbf{1 . 6 2}) & \mathbf{1 . 4 4}(\mathbf{1 . 0 8} \mathbf{1 . 9 1}) & 1.09(0.67,1.79) \\ \mathbf{1 . 0 2}(\mathbf{1 . 0 1}, \mathbf{1 . 0 4}) & & & & \end{array}$

$1.01,1.04)$

Normoweight Overweight n (\%)

n (\%)

$\begin{array}{ll}746(48.2) & 1234(47.3) \\ 801(51.8) & 1373(52.7) \\ 1 \text { (ref.) } & 1.04(0.91,1.19) \\ 1 \text { (ref.) } & 1.04(0.92,1.18) \\ 0.99(0.98,1.01) & \end{array}$

$915(59.2)$

$1473(56.6)$

631 (40.8)

1 (ref.)

$1129(43.4)$

$1.11(0.98,1.26)$

479 (43.7)

1 (ref.)

$1.12(0.98,1.27)$

$0.99(0.98,1.01)$

966 (62.6)

578 (37.4)

1 (ref.)

947 (36.3)

1 (ref.)

$0.95(0.84,1.09)$

$1.01(0.99,1.03)$

$1288(83.2)$
261 (16.8)
1 (ref.)
1 (ref.)

2094 (80.4)

512 (19.6)

862 (78.2)

$1.21(1.02,1.42)$

$1.12(0.95,1.33)$

$1.02(1.00,1.04)$

$930(60.2)$
$614(39.8)$
1 (ref.)
1 (ref.)

1633 (62.6)

$0.90(0.80,1.03)$

$0.96(0.95,0.98)$

$\begin{array}{ll}1486(96.1) & 2486(95.6) \\ 61(3.9) & 115(4.4) \\ 1 \text { (ref.) } & 1.13(0.82,1.54) \\ 1 \text { (ref.) } & 1.10(0.80,1.52) \\ 1.00(0.97,1.03) & \end{array}$

515 (46.8)

585 (53.2)

$1.06(0.91,1.24)$

$1.04(0.89,1.22)$

618 (56.3)

$1.12(0.96,1.31)$

106 (39.3)

$0.94(0.72,1.22)$

$1.12(0.95,1.31)$

$0.91(0.69,1.20)$

639 (58.1)

$150(55.1)$

55 (64.0)

461 (41.9)

133 (44.9)

$1.21(1.03,1.41)$

$1.36(1.05,1.76)$

$31(36.0)$

$1.16(0.99,1.37)$

$1.29(0.98,1.70)$

$0.94(0.60,1.48)$

211 (77.6)

69 (80.2)

$61(22.4)$

17 (19.8)

$1.37(1.13,1.67)$

$1.43(1.04,1.95)$

$1.22(0.70,2.10)$

696 (63.3)

403 (36.7)

$0.88(0.75,1.03)$

$0.85(0.72,1.00)$
$263(96.7)$

9 (3.3)

$1.41(0.98,2.04)$

$0.83(0.41,1.70)$

$0.75(0.36,1.54)$

$1.36(0.94,1.98)$
$70(81.4)$

16 (18.6)

$0.61(0.46,0.81) \quad 0.35(0.20,0.60)$

$0.58(0.43,0.77) \quad 0.33(0.19,0.58)$
$3472(61.8)$

$2150(38.2)$

$3233(57.7)$

$2367(42.3)$

$2676(47.7)$

$2936(52.3)$

Total values

n (\%) n (\%)

$0.51(0.31,0.83)$

$0.52(0.31,0.86)$

$0.93(0.53,1.65)$

4524 (80.6)

$1091(19.4)$

$3522(62.8)$

$2086(37.2)$
83 (96.5)

3 (3.5)

5353 (95.6)

$248(4.4)$ 
Table 5 Complications and discomfort during hospitalisation and at home related to breastfeeding according to maternal BMI (Continued)

\begin{tabular}{|c|c|c|c|c|c|c|}
\hline \multicolumn{7}{|c|}{$\begin{array}{l}\text { Delayed milk production } \\
\text { (after the 5th day of delivery)? }\end{array}$} \\
\hline No & $1406(90.8)$ & $2343(89.9)$ & $959(87.2)$ & $233(86.0)$ & $71(82.6)$ & $5012(89.3)$ \\
\hline Yes & $142(9.2)$ & $262(10.1)$ & $141(12.8)$ & $38(14.0)$ & $15(17.4)$ & $598(10.7)$ \\
\hline OR CI 95\% & 1 (ref.) & $1.11(0.89,1.37)$ & $1.46(1.14,1.86)$ & $1.62(1.10,2.37)$ & $2.09(1.17,3.75)$ & \\
\hline${ }^{b} \mathrm{AOR}$ CI 95\% & 1 (ref.) & $1.08(0.86,1.35)$ & $1.31(1.01,1.71)$ & $1.43(0.94,2.16)$ & $1.83(0.96,3.50)$ & \\
\hline${ }^{\mathrm{b}} \mathrm{AOR}$ Cl 95\% Total & \multicolumn{6}{|c|}{$1.03(1.01,1.05)$} \\
\hline \multicolumn{7}{|l|}{$\begin{array}{l}\text { The baby not gaining } \\
\text { enough weight? }\end{array}$} \\
\hline No & $1180(76.1)$ & $1976(76.0)$ & $847(77.0)$ & $188(69.4)$ & $57(66.3)$ & $4248(75.7)$ \\
\hline Yes & $371(23.9)$ & $624(24.0)$ & $253(23.0)$ & $83(30.6)$ & $29(33.7)$ & $1360(24.3)$ \\
\hline OR CI 95\% & 1 (ref.) & $1.00(0.87,1.16)$ & $0.95(0.79,1.14)$ & $1.40(1.06,1.86)$ & $1.62(1.02,2.57)$ & \\
\hline${ }^{b} A O R C I 195 \%$ & 1 (ref.) & $1.00(0.86,1.16)$ & $0.93(0.77,1.13)$ & $1.37(1.01,1.84)$ & $1.79(1.10,2.90)$ & \\
\hline${ }^{\mathrm{b}} \mathrm{AOR}$ Cl 95\% Total & $1.01(0.99,1$. & & & & & \\
\hline \multicolumn{7}{|c|}{ 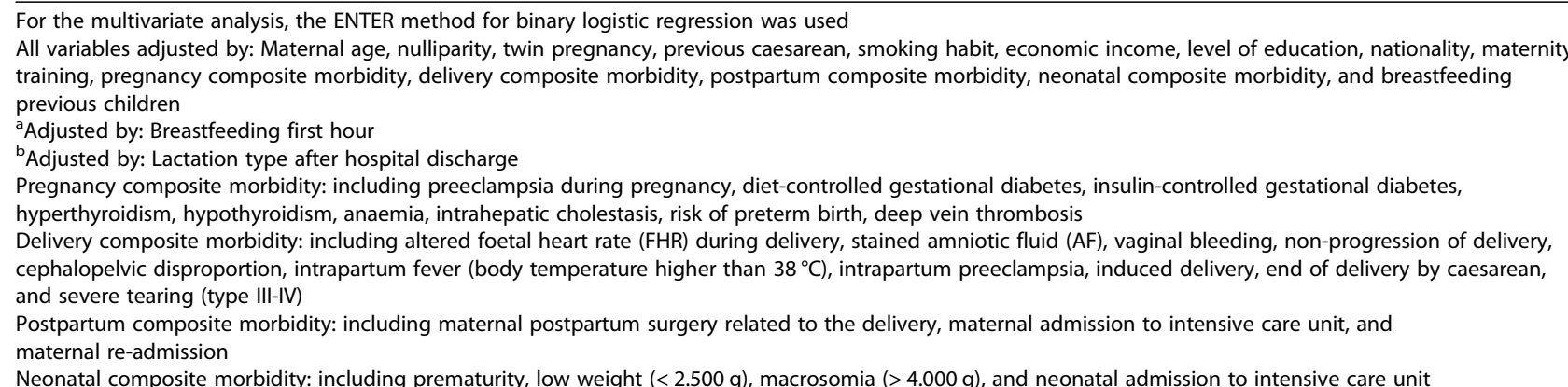 } \\
\hline
\end{tabular}

the intake takes longer, so as to stimulate milk production [28].

As for the limitations, a bias of information in considered unlikely as the data collected and the way in which the possible responses were presented did not require a high level of education. In this regard, the questions were asked in a basic and simple way, and any participant could understand them regardless of their level of education. It is not possible to completely rule out the confusion bias inherent in observational studies, but it has been controlled by using a multivariate analysis technique. As limitation, it is also worth mentioning that the proportion of foreign women and level of education is not representative of the whole present Spanish society, so these population groups were difficult to extrapolate. On the other hand, BMI was self-reported by women, and this was based on their clinical reports, according to the women's own understanding of their clinical data, so there is a possibility that some women were not fully honest when giving their answers. However, several authors have observed that self-reported data on BMI can be successfully used in epidemiological studies as the differences are minimal among young populations and with a high level of education, as are the women in our sample [29-31]. Other limitations were the absence of underweight women among our sample and the impossibility of separating preeclampsia and chronic hypertension as different pathologies, as also happened with diabetes type I. Furthermore, the definition of the outcome variables such as hypertension or anaemia was based on the diagnosis recorded in the medical records and not on cut-off points of measurements or analytical values.

Among the main strengths of the study, the large sample size and the scarcity of studies focused on specific aspects of breastfeeding such as starting in the first hour or skin-to-skin contact can be highlighted. On the other hand, the prevalence of obesity in our study was similar to other studies, such as that by Parveen et al. [2], who observed a prevalence of obesity of $31.6 \%$, and the one by Melchor et al. [32], with a prevalence of $18.4 \%$. Because of this, we believe that our sample provides an adequate representation of women with high BMIs.

\section{Conclusions}

Finally, we can conclude that women with higher BMI are more likely to give up breastfeeding and have problems associated with it. More specifically, these women are less likely to start skin-to-skin contact, breastfeeding within the first hour, exclusive breastfeeding at hospital 
discharge, and they also show a higher perception of late lactogenesis. As breastfeeding is considered a public health measure, mechanisms to support these women should be put in place both during their hospital admission and within the community. An approach to this situation from a qualitative point of view could be interesting so as to know more about the causes and enable the development of more effective intervention strategies.

\section{Supplementary information}

Supplementary information accompanies this paper at https://doi.org/10. 1186/s13006-020-00298-5.

Additional file 1. Questionnaire.

Additional file 2. List of associations that have collaborated in the distribution of the questionnaire.

\section{Conflict of interest}

The authors declare no conflict of interest.

\section{Authors' contributions}

Conceptualization, ABC and JRA.; Methodology, IOE and AHM; Formal Analysis, JGS and ABC; Writing-Original Draft Preparation, $A B C$ and JRA; Writing-Review \& Editing, IOE and AHM; Supervision, JRA and AHM; Project Administration, JGS; The authors read and approved the final manuscript.

\section{Funding}

None received.

\section{Availability of data and materials}

Not applicable.

\section{Ethics approval and consent to participate}

This study has received the approval of the Clinical Research Ethics Committee of Alcázar de San Juan, in Spain, with protocol number 92-C.

\section{Consent for publication}

The authors give their consent for publication.

\section{Competing interests}

Not applicable.

\section{Author details}

${ }^{1}$ Department of Obstetrics \& Gynaecology, Talavera de la Reina, 45600 Toledo, Spain. ${ }^{2}$ Department of Sociology, Social Work and Public Health, University of Huelva, 21071 Huelva, Spain. ${ }^{3}$ Safety and Health Postgraduate Programme, Universidad Espíritu Santo, 091650 Guayaquil, Ecuador. ${ }^{4}$ Department of Nursing, Ciudad Real Nursing School, University of Castilla-La Mancha, 13071 Ciudad Real, Spain. ${ }^{5}$ Department of Obstetrics \&

Gynaecology, Alcázar de San Juan, 13600 Ciudad Real, Spain.

Received: 23 December 2019 Accepted: 2 June 2020

Published online: 15 June 2020

\section{References}

1. Galán SM, Hernández ÁS, Zúñiga IV, Löpez Criado MS, Lloréns AP, José Luis Gallo V. Abnormal maternal body mass index and obstetric and neonatal outcome. J Matern Neonatal Med. 2012;25(3):308-12.

2. Parveen $T$, Hameed $F$, Kahn JA. The impact of maternal obesity on neonatal Apgar score and neonatal intensive care unit admissions in a tertiary care hospital. Pak J Surj. 2018;34(2):161-5.

3. Zhu T, Tang J, Zhao F, Qu Y, Mu D. Association between maternal obesity and offspring Apgar score or cord $\mathrm{pH}$ : a systematic review and metaanalysis. Sci Rep. 2015:5:18386.

4. Lepe M, Bacardí Gascón M, Castañeda-González LM, Pérez Morales ME, Jiménez CA. Effect of maternal obesity on lactation: systematic review. Nutr Hosp. 2011;26(6):1266-9.
5. He X, Zhu M, Hu C, Tao X, Li Y, Wang Q, et al. Breast-feeding and postpartum weight retention: a systematic review and meta-analysis. Public Health Nutr. 2015;18(18):3308-16.

6. Simko M, Totka A, Vondrova D, Samohyl M, Jurkovicova J, Trnka M, et al. Maternal body mass index and gestational weight gain and their association with pregnancy complications and perinatal conditions. Int J Environ Res Public Health. 2019;6(10):E1751.

7. Flores TR, Mielke Gl, Wendt A, Nunes BP, Bertoldi AD. Prepregnancy weight excess and cessation of exclusive breastfeeding: a systematic review and meta-analysis. Eur J Clin Nutr. 2018;72(4):480-8.

8. Boudet-Berquier J, Salanave B, Desenclos JC, Castetbon K. Association between maternal prepregnancy obesity and breastfeeding duration: data from a nationwide prospective birth cohort. Matern Child Nutr. 2018;14(2):e12507.

9. Preusting I, Brumley J, Odibo L, Spatz DL, Louis JM. Obesity as a predictor of delayed Lactogenesis II. J Hum Lact. 2017;33(4):684-91.

10. Lyons S, Currie S, Peters S, Lavender T, Smith DM. The association between psychological factors and breastfeeding behaviour in women with a body mass index (BMI) $\geq 30 \mathrm{~kg} \mathrm{~m}-2$ : a systematic review. Obes Rev. 2018;19(7): 947-59.

11. Marshall NE, Lau B, Purnell JQ, Thornburg KL. Impact of maternal obesity and breastfeeding intention on lactation intensity and duration. Matern Child Nutr. 2019;15(2):e12732.

12. Wojcicki JM. Maternal prepregnancy body mass index and initiation and duration of breastfeeding: a review of the literature. J Women's Health. 2011;20(3):341-7.

13. Ramón-Arbués $E$, Martínez-Abadía B, Gracía-Tabuenca T, Yuste-Gran C Pellicer-García B, Juárez-Vela R, et al. Nutr Hosp. 2019;36(1):51-9.

14. Hernáez Á, Zomeño MD, Dégano IR, Pérez-Fernández S, Goday A, Vila J, et al. Excess weight in Spain: current situation, projections for 2030, and estimated direct extra cost for the Spanish health system. Revista Rev Esp Cardiol. 2019:72(11):916-24.

15. Peduzzi P, Concato J, Kemper E, Holford TR, Feinstein AR. A simulation study of the number of events per variable in logistic regression analysis. J Clin Epidemiol. 1996;49(12):1373-9.

16. Athukorala C, Rumbold AR, Willson KJ, Crowther CA. The risk of adverse pregnancy outcomes in women who are overweight or obese. BMC Pregnancy Childbirth. 2010;10:56

17. Hadley EE, Discacciati A, Costantine MM, Munn MB, Pacheco LD, Saade GR, et al. Maternal obesity is associated with chorioamnionitis and earlier indicated preterm delivery among expectantly managed women with preterm premature rupture of membranes. J Matern Neonatal Med. 2019; 32(2):271-8.

18. Masho SW, Cha S, Morris MR. Prepregnancy obesity and breastfeeding noninitiation in the United States: an examination of racial and ethnic differences. Breastfeed Med. 2015;10(5):253-62.

19. Amir LH, Donath $\mathrm{S}$. A systematic review of maternal obesity and breastfeeding intention, initiation and duration. BMC Pregnancy Childbirth. 2007;7:9.

20. Mehta UJ, Siega-Riz AM, Herring AH, Adair LS, Bentley ME. Pregravid body mass index, psychological factors during pregnancy and breastfeeding duration: is there a link? Matern Child Nutr. 2012:8(4):423-33.

21. Ruhstaller KE, Elovitz MA, Stringer M, Epperson CN, Durnwald CP. Obesity and the association with maternal mental health symptoms. J Matern Neonatal Med. 2017;30(16):1897-901.

22. Dutton GR, Lewis TT, Durant N, Halanych J, Kiefe Cl, Sidney S, et al. Perceived weight discrimination in the CARDIA study: differences by race, sex, and weight status. Obesity. 2014;22(2):530-6.

23. Swanson V, Keely A, Denison FC. Does body image influence the relationship between body weight and breastfeeding maintenance in new mothers? Br J Health Psychol. 2017;22(3):557-76.

24. Bigman G, Wilkinson AV, Homedes N, Pérez A. Body image dissatisfaction, obesity and their associations with breastfeeding in Mexican women, a cross-sectional study. Matern Child Health J. 2018;22(12):1815-25.

25. Tavoulari EF, Benetou V, Vlastarakos PV, Andriopoulou E, Kreatsas G, Linos A Factors affecting breast-feeding initiation in Greece: what is important? Midwifery. 2015;31(2):323-31.

26. Kuhn NJ. Progesterone withdrawal as the lactogenic trigger in the rat. 1969 Mammary Gland Biol Neoplasia. 2009;14(3):327-42

27. Rasmussen KM, Kjolhede CL. Prepregnant overweight and obesity diminish the prolactin response to suckling in the first week postpartum. Pediatrics. 2004;113(5):e465-71. 
28. Kent JC, Prime DK, Garbin CP. Principles for maintaining or increasing breast Milk production. JOGNN. 2012;41(1):114-21.

29. Pursey K, Burrows TL, Stanwell P, Collins CE. How accurate is web-based self-reported height, weight, and body mass index in young adults. J Med Internet Res. 2014;16(1):e4.

30. Olfert MD, Barr ML, Charlier CM, Famodu OA, Zhou W, Mathews AE, et al. Self-reported vs. measured height, weight, and BMl in young adults. Int J Environ Res Public Health. 2018;15(10):2216.

31. Savane FR, Navarrete-Muñoz EM, de la Hera MG, Gimenez-Monzo D, Gonzalez-Palacios S, Valera-Gran D, et al. Validez del peso y talla autoreferido en población universitaria y factores asociados a las discrepancias entre valores declarados y medidos. Nutr Hosp. 2013;28(5):1633-8.

32. Melchor I, Burgos J, Del Campo A, Aiartzaguena A, Gutiérrez J, Melchor JC. Effect of maternal obesity on pregnancy outcomes in women delivering singleton babies: a historical cohort study. J Perinat Med. 2019;47(6):625-30.

\section{Publisher's Note}

Springer Nature remains neutral with regard to jurisdictional claims in published maps and institutional affiliations.

Ready to submit your research? Choose BMC and benefit from:

- fast, convenient online submission

- thorough peer review by experienced researchers in your field

- rapid publication on acceptance

- support for research data, including large and complex data types

- gold Open Access which fosters wider collaboration and increased citations

- maximum visibility for your research: over $100 \mathrm{M}$ website views per year

At BMC, research is always in progress.

Learn more biomedcentral.com/submissions 